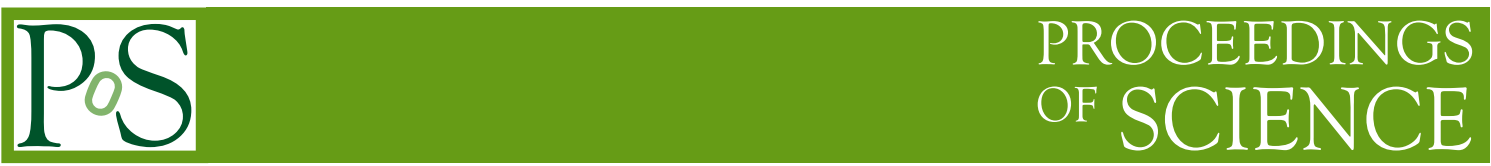

\title{
The LHCf experiment
}

K. Noda ${ }^{* a}$, O. Adriani ${ }^{b c}$, L. Bonechi ${ }^{b}$, M. Bongi ${ }^{b}$, G. Castellini ${ }^{b c}$, R. D'Alessandro ${ }^{b c}$, K. Fukatsu ${ }^{d}$, M. Haguenauer ${ }^{e}$, Y. Itow ${ }^{d f}$, K. Kasahara ${ }^{g}$, K. Kawade ${ }^{d}$, D. Macina ${ }^{h}$,

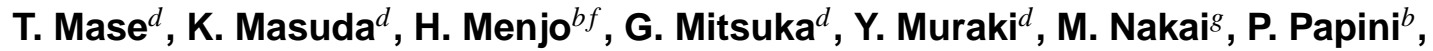
A.-L. Perrot ${ }^{h}$, S. Ricciarini ${ }^{b i}$, T. Sako ${ }^{d f}$, Y. Shimizu ${ }^{g}$, K. Suzuki $^{d}$, T. Suzuki ${ }^{g}$, K. Taki $^{d}$, T. Tamura ${ }^{j}$, S. Torii $^{g}$, A. Tricomi ${ }^{a k}$ and W. C. Turner ${ }^{l}$

${ }^{a}$ INFN Section of Catania, Italy

${ }^{b}$ INFN Section of Florence, Italy

${ }^{c}$ University of Florence, Italy

${ }^{d}$ Solar-Terrestrial Environment Laboratory, Nagoya University, Nagoya, Japan

${ }^{e}$ Ecole-Polytechnique, Palaiseau, France

${ }^{f}$ Kobayashi-Maskawa Institute for the Origin of Particles and the Universe, Nagoya University, Nagoya, Japan

${ }^{g}$ RISE, Waseda University, Japan

${ }^{h}$ CERN, Switzerland

${ }^{i}$ Centro Siciliano di Fisica Nucleare e Struttura della Materia, Catania, Italy

${ }^{j}$ Kanagawa University, Japan

${ }^{k}$ University of Catania, Italy

${ }^{l}$ LBNL, Berkeley, California, USA

E-mail: koji.noda@ct.infn.it

LHCf is a collider experiment dedicated to cosmic-ray physics. It measures the energy spectrum of very forward particles generated in pp collisions in LHC. Its aim is to minimize systematic errors in air-shower simulations used in ultra-high-energy cosmic-ray experiments like the Pierre Auger Observatory. In this paper we briefly summarize the current status of the experiment: DAQ, analyses, and works for foreseen detector upgrade.

VI European Summer School on Experimental Nuclear Astrophysics, ENAS 6

September 18-27, 2011

Acireale Italy

\footnotetext{
* Speaker.
} 


\section{Introduction}

Cosmic rays with energy above $10^{15} \mathrm{eV}$ have been detected on the Earth since several decades ago, but there still remain many uncertainties in understanding of their properties. In particular, the properties of cosmic rays beyond $10^{18} \mathrm{eV}$, called ultra-high-energy cosmic rays (UHECRs), have not been clarified yet. We do not know much about their chemical composition, the propagation mechanism, and thus the astrophysical origin. One reason is that they can be detected indirectly only through a particle cascade called air-shower, and that we must always care for systematic errors caused by the air-shower development.

If we try to understand the cosmic rays in a step-by-step way from the Earth side, we must understand first what the cosmic rays are, i.e., the chemical composition. For its determination, the maximum depth of the air-shower development is generally used. Recently, a measurement by Pierre Auger Observatory suggests that the chemical composition of UHECR has a gradual transition from a light chemical composition (proton) to a heavy one (iron) [1]. However, this result is different from a result by the HiRes experiment [2]. Furthermore, the depth of the shower maximum is severely dependent on the hadronic interaction models used in Monte Carlo simulations for the air-shower development. The models are based on on the perturbative QCD for hard part and on the Regge Field theory for soft part but they treat the non-linear effects for soft and semi-hard part only in phenomenology. Therefore, in order to reduce the uncertainty in the discussion of the chemical composition, we must reduce first the model dependence with experimental data obtained in colliders like the Large Hadron Collider (LHC).

There are several key quantities for the air-shower development. It starts with the first interaction of the cosmic ray and a nucleus of the atmosphere, where the inelastic cross section of the first interaction is important. In LHC the TOTEM experiment has already measured the inelastic cross section [3]. Then, the energy of the cosmic ray is transferred inelastically to secondary particles like baryons and mesons. This secondary particles emitted at forward angles produce more particles in cascade. Thus, the amount of transferred energy (inelasticity) and the energy spectrum of the forward particles are important here. The latter is the main goal of the LHCf experiment [5]. It measures the spectra of very forward neutral particles (photons, neutral pions and neutrons) emitted in high energy collisions in LHC. As for the spectrum of forward pions, available data with the highest energy is that for neutral pions by the UA7 experiment [4] in SPS, whose collision energy corresponds to $10^{14} \mathrm{eV}$ in cosmic-ray energy. On the other hand, the LHCf experiment is designed to obtain data with a collision energy of $14 \mathrm{TeV}$, corresponding to $10^{17} \mathrm{eV}$ in cosmic-ray energy. Thus, it will be expected to reduce the uncertainty of the model dependence close to the UHECR energy.

\section{The LHCf detector}

LHCf is installed at the CERN Large Hadron Collider in Geneva, Switzerland. The LHCf detector is located at $140 \mathrm{~m}$ away from Interaction Point 1 (IP1; the ATLAS site) and at zero degree collision angle. The detector is installed in the instrumentation slots of the neutral particle absorbers (TAN). Inside the TAN the beam vacuum chamber makes a Y-shaped transition from a single beam tube facing the IP to two separate beam tubes joining to the arcs of LHC. The TAN instrumentation 

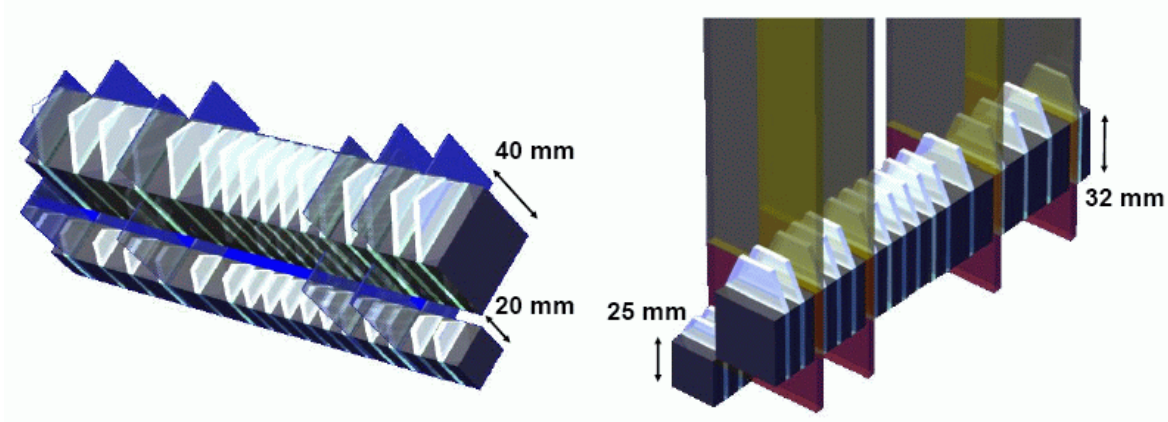

Figure 1: Schematic view of the LHCf detectors (Arm1 on the left, Arm2 on the right). Plastic scintillators (light blue color) are interleaved with tungsten blocks (dark gray color). Four position sensitive layers (scintillating fibers in Arm1, dark blue color; silicon micro-strip detectors in Arm2, purple color) are distributed in each calorimeter.

slot is in the crotch of the Y. Charged secondary particles from IP are swept aside by the inner beam separation dipole before reaching TAN, so only neutral particles are incident on the LHCf detectors. This location covers the pseudo-rapidity range from 8.4 to infinity. This instrumentation slot exists in each of both sides of IP1, thus we have two detectors located on opposite sides of IP1, named Arm1 and Arm2.

The overall concepts for the two Arms are the same (Fig. 11). Each of the two LHCf detectors consists of two small sampling calorimeters and four position sensitive layers inserted into the calorimeter layers. The calorimeter is made of 16 plastic scintillators sandwiched by tungsten absorbers, and it has a total length equivalent to 44 radiation lengths, and 1.55 interaction length. We call the calorimeters small \& large towers. The four position sensitive layers are distributed among the layers of the calorimeters for determining the transverse shower positions. Arm1 utilizes scintillating fibers as the position-sensitive layers, while Arm2 uses silicon ( $\mathrm{Si}$ ) microstrip sensors. In addition the geometrical configurations of the two towers for each Arm are different for the purposes of redundancy and consistency check. In front of each detector, a Front Counter made of plastic scintillators is inserted. It provides useful trigger information by covering a larger aperture than the calorimeters. Many additional details of both Arms can be found in [5].

The detector can identify photons with the two calorimeters. It measures their energy spectrum beyond $100 \mathrm{GeV}$ with less than $5 \%$ energy resolution, and their incident position with $0.2 \mathrm{~mm}$ position resolution. If a photon is detected in each of the two calorimeters at the same time, it is expected to be reconstructed as an event from a neutral pion. This is the main target of the LHCf detector. Measured spectrum of neutral pions can be used to discriminate among the hadronic interaction models. Also, hadronic showers of high-energy neutrons can be measured with energy resolution of about $30 \%$.

\section{Data acquisition}

The current LHCf detector was not designed to be a radiation hard detector. That is because the model discrimination requires just a short period during the early phase of the LHC commissioning before the high luminosity operations. For the LHC operation in 2009 and 2010, we have obtained 

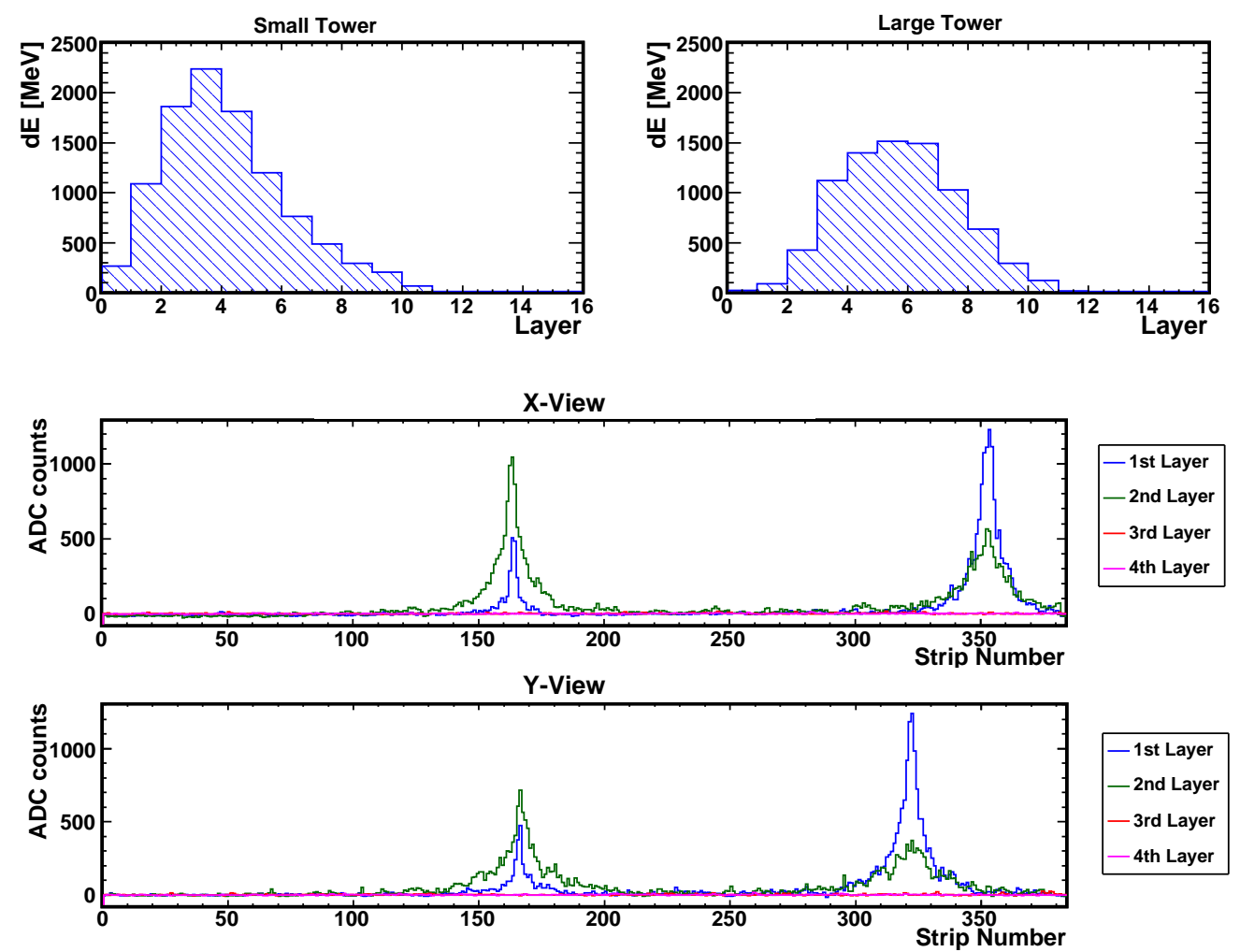

Figure 2: An example of data taken during 2010 operation [7] (a candidate for detection of two photons from the decay of a neutral pion). The upper two panels show the transition curves taken by the two calorimeters; The left is by the small tower, while the right is by the large tower. The lower two panels are the data from the Si microstrip sensors; The upper is the four $\mathrm{x}$-view data, while the lower is the four $\mathrm{y}$-view data. The identifiable peaks are from the 1 st and 2 nd layers, while the data from the $3 \mathrm{rd}$ and 4th layers are almost zero.

data for pp collision with energies of $\sqrt{s}=0.9 \& 7 \mathrm{TeV}$. The accumulated numbers of events are about $100 \mathrm{k}$ showers at $0.9 \mathrm{TeV}$, and about $400 \mathrm{M}$ showers at $7 \mathrm{TeV}$, corresponding to about $1 \mathrm{M}$ neutral pion events, for each of the two Arms. After the operation for several months, the detectors were removed from the slot in 2010.

In Fig. 2. we show an example of a candidate of a neutral pion event, i.e., one photon for each tower, obtained by Arm2. We can see sharp peaks from single photon events detected by the first two layers of the Si microstrip sensors. With the information we can reconstruct the invariant mass of the photon pairs. It is expected to be around the mass of the neutral pion $(135 \mathrm{MeV})$ and will be used for an energy calibration. We have also obtained the energy spectra of the neutral pions for both of the Arms. A preliminary result can be seen in [6.

\section{Photon analysis}

The first physics result from the LHCf experiment has been published in 2011 [7]. It is dedicated to measurement of the single photon spectrum. Events by neutral hadrons are removed by a simple criteria based on the longitudinal development of the showers, obtained by the scintillators. The energy of the remaining photons is determined from the same information on the light 
produced in the scintillators. We applied corrections to it; for non-uniformity of light collection efficiency, and for particles leaking out of the edges of the calorimeter towers. In this corrections we used the lateral positions of showers determined with the information by the position sensitive layers. The information by the position sensitive layers is also used to exclude 'multi-hit' events that has more than one showers inside the same tower. The two Arms have different geometrical configurations, thus in this analysis we have selected a common region for each tower in order to combine the two spectra without any acceptance correction. The region is pseudo-rapidity $\eta>$ 10.94 and azimuthal range $\Delta \phi=360^{\circ}$ for the small towers, while $8.81<\eta<8.99$ and $\Delta \phi=20^{\circ}$ for the large towers. Further details about the analysis are found in [7].

Figure 3 shows the single-photon energy spectra obtained from a data set taken in pp collisions at $\sqrt{ } s=7 \mathrm{TeV}$ in 2010. During the period for the data set, the integrated luminosity is estimated to be $\int L d t=0.68 \mathrm{nb}^{-1}$ and $0.53 \mathrm{nb}^{-1}$ for Arm1 and Arm2, respectively. Multiplying it with an assumed inelastic cross section $\sigma_{\text {ine }}=71.5 \mathrm{mb}$, we derived the number of inelastic collisions, $N_{\text {ine }}$, on the vertical axis. The black points are the energy spectra obtained by the combination of the two Arms, and they are compared with results predicted by MC simulations using different models: DPMJET 3.04[8], QGSJET II-03 [9], SIBYLL 2.1 [10], EPOS 1.9[11] and PYTHIA 8.145[12]13]. Left and right panels refer to the selected regions as mentioned before.

As a result, we see that none of the model predictions nicely describe the LHCf data in the whole energy range from $100 \mathrm{GeV}$ to $3.5 \mathrm{TeV}$. In particular, there is a big discrepancy in the high energy region. Discussions about this result with the model developers have been already started. Improvements of the hadron interaction models are expected in near future.

\section{Future prospects}

Now we are working on the following analyses for topics such as neutral pions, $900 \mathrm{GeV}$ photons, and Pt distribution of photons and hadron spectra at $7 \mathrm{TeV}$ collisions. At the same time, we are preparing for foreseen data acquisitions with the proton-ion collision in 2012, and the p-p collisions at $\sqrt{ } s=14 \mathrm{TeV}$ in 2014. A higher luminosity is expected for the $14 \mathrm{TeV}$ runs than that in the $7 \mathrm{TeV}$ runs. Thus we are planning a hardware upgrade of the plastic scintillators and the scintillating fibers to ones made of $\mathrm{Gd}_{2} \mathrm{SiO}_{5}$ (GSO), which are more radiation-hard than the plastic scintillators. The radiation hardness of the GSO scintillator was measured in a test beam, and we confirmed that it has properties good enough for our use [14].

Another upgrade is planned for the Arm2 detector. The current configuration of the Si microstrip sensors distributed in the plastic scintillators are not optimized for an energy reconstruction using the Si sensors. If we change the configuration, an improvement of the energy resolution only with the Si is expected by a MC study, to be less than $10 \%$. However, the Si sensors were originally not considered as a calorimeter, thus we require additional works for them. The first is the gain calibration using the test beam data and an MC simulation for the same configuration. We have obtained a gain factor from ADC counts to the energy deposit on the Si sensors. Then the incident particle energy can be reconstructed from the energy deposit, using a function obtained by a simulation for LHC configuration. A preliminary result with a MC data set shows that the energy resolution for photons is $\sim 15 \%$ up to $1.5 \mathrm{TeV}$ [15]. When the Si sensor is used as a calorimeter, 

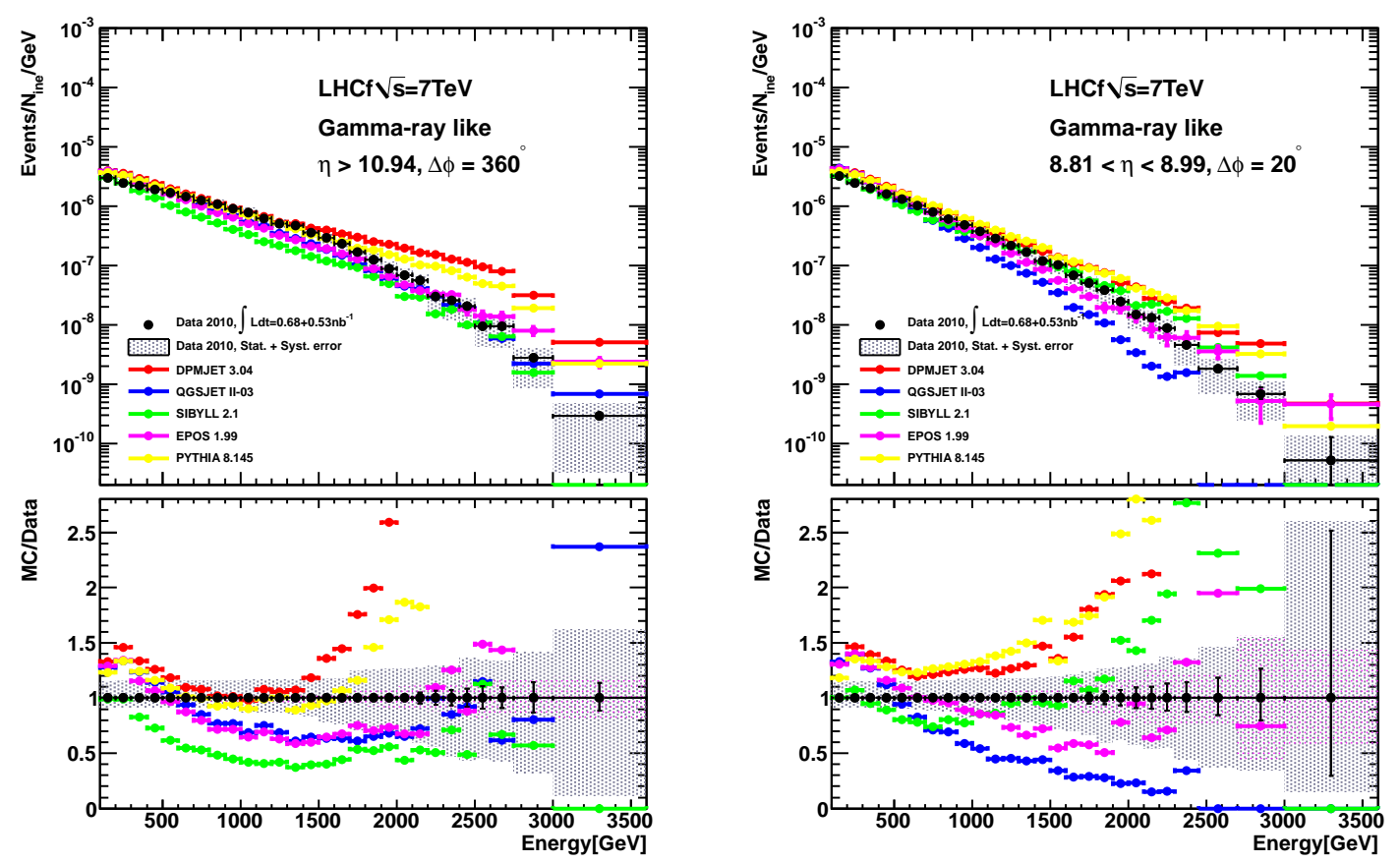

Figure 3: Comparison between the measured single-photon energy spectra (black dots) and the predictions of the following MC codes: DPMJET 3.04 (red), QGSJET II-03 (blue), SIBYLL 2.1 (green), EPOS 1.99 (magenta) and PYTHIA 8.145 (yellow), taken from [7]. Top panels show the spectra and bottom panels show the ratio of MC results to experimental data. Left and right panels refer to different pseudo-rapidity ranges. Error bars show the statistical error and gray shaded areas the systematic error for experimental data. Magenta shaded areas indicate the statistical error associated to MC simulations.

it would help not only the calorimetry of the scintillators, but also a separation of the 'multi-hit' events, which could not be resolved only by the scintillators.

\section{Summary}

LHCf is a collider experiment dedicated for the cosmic-ray physics. It measures the energy spectrum of very forward particles generated in pp collisions in LHC. Its aim is to minimize systematic errors in air-shower simulations used in ultra-high-energy cosmic-ray experiments. In this paper we briefly surveyed our detector and the current status of the experiment. We have already done the data acquisition for pp collisions at $\sqrt{ } s=7 \mathrm{TeV}$, and removed the detectors. The obtained photon spectra are not well described by the MC expectations, especially in high energies. Now we are working for following analyses and foreseen detector upgrade. The prospects about the hardware upgrade were also shown in this paper.

\section{References}

[1] J. Abraham et al. (Auger Collaboration), Measurement of the Depth of Maximum of Extensive Air Showers above $10^{18} \mathrm{eV}$, Physical Review Letters, 104 (2010) 091101. 
[2] R. U. Abbasi et al. (HiRes Collaboration), Indications of Proton-Dominated Cosmic-Ray Composition above 1.6 EeV, Physical Review Letters, 104 (2010) 161101.

[3] G. Antchev et al. (TOTEM Collaboration), First measurement of the total proton-proton cross section at the LHC energy of $\sqrt{ } s=7 \mathrm{TeV}$, Europhysics Letters, 96 (2011) 21002.

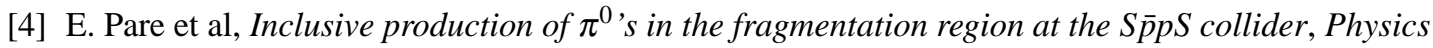
Letter B, 242 (1990) 531.

[5] O. Adriani et al. (LHCf Collaboration), The LHCf detector at the CERN Large Hadron Collider, JINST, 3 (2008) S08006.

[6] H. Menjo et al. (LHCf Collaboration), Energy spectrum of neutral pion at LHC proton-proton collisions measured by the LHCf experiment (HE1.4, 1335), Proceedings of the 32nd International Cosmic Ray Conference (2011) in press.

[7] O. Adriani et al. (LHCf Collaboration), Measurement of zero degree single photon energy spectra for $\sqrt{ } s=7$ TeV proton-proton collisions at LHC, Physics Letter B, 703 (2011) 128.

[8] F. W. Bopp et al., Antiparticle to particle production ratios in hadron-hadron and d-Au collisions in the DPMJET-III Monte Carlo model, Physical Review C, 77 (2008) 014904.

[9] S. Ostapchenko, Nonlinear screening effects in high energy hadronic interactions, Physical Review D, 74 (2006) 014026.

[10] E.-J. Ahn et al., Cosmic ray interaction event generator SIBYLL 2.1, Physical Review D, 80 (2009) 094003.

[11] K. Werner et al., Parton ladder splitting and the rapidity dependence of transverse momentum spectra in deuteron-gold collisions at the BNL Relativistic Heavy Ion Collider, Physical Review C, 74 (2006) 044902.

[12] T. Sjöstand et al., PYTHIA 6.4 physics and manual, JHEP, 05 (2006) 026.

[13] T. Sjöstand et al., A brief introduction to PYTHIA 8.1, Computer Physics Communications, 178 (2008) 852 .

[14] K. Kawade et al., Study of radiation hardness of $\mathrm{Gd}_{2} \mathrm{SiO}_{5}$ scintillator for heavy ion beam, JINST, 6 (2011) T09004.

[15] K. Noda et al. (LHCf Collaboration), Data analysis of the LHCf Si microstrip sensors (HE3.1, 421), Proceedings of the 32nd International Cosmic Ray Conference (2011) in press. 